Sādhanā Vol. 40, Part 4, June 2015, pp. 1117-1137. (C) Indian Academy of Sciences

\title{
Runtime buffer management to improve the performance in irregular Network-on-Chip architecture
}

\author{
UMAMAHESWARI $\mathrm{S}^{1, *}$, MEGANATHAN $\mathrm{D}^{2}$ and \\ RAJA PAUL PERINBAM J ${ }^{3}$ \\ ${ }^{1}$ Department of Information Technology, Anna University, MIT Campus, Chennai \\ 600044 , India \\ ${ }^{2}$ Department of Electronics, Anna University, MIT Campus, Chennai 600 044, India \\ ${ }^{3}$ Department of ECE, KCG College of Technology, Chennai 600 097, India \\ e-mail: uma_sai@annauniv.edu; meganathan_phd@annauniv.edu; \\ rperinbam@yahoo.com
}

MS received 1 November 2014; revised 22 January 2015; accepted 6 February 2015

\begin{abstract}
This paper presents a heterogeneous adaptable router to reduce latency in irregular mesh Network-on-Chip (NoC) architectures. Regular mesh-based NoC architecture may become irregular due to variable sized IPs and needs new routing algorithms to ensure throughput. Therefore, an irregular NoC mesh is considered and an adaptive algorithm is used for routing. The performance measures such as throughput, latency, and bandwidth are defined at design time to guarantee the performance of NoC. However, if the application has to change its communication pattern, parameters set at design time (say buffer size) may result in large area and power consumption or increased latency. Routers with large input buffers improve the efficiency of NoC communication, but they incur excessive power dissipation and hardware overheads. Routers with small buffers reduce power consumption, but result in high latency. In the proposed NoC router, input buffers can be dynamically allocated, thereby, latency can be reduced. In a $4 \times 4$ irregular mesh NoC with a buffer depth of 4 slots, $20 \%$ reduction in latency and $9 \%$ increase in throughput are attained using dynamic buffer allocation. An $8 \times 8$ irregular mesh NoC with the proposed router is exposed to the synthetic traffics like uniform, bit complement, tornado and hotspot traffics and it offered a $30.42 \%$ reduction in overall average latency and $18.33 \%$ increase in overall saturation throughput. The proposed router outperformed the static router by $22.63 \%$ less average latency for E3S benchmark applications. For the same performance, maximum of 55\% reduction in buffer requirement and 53\% less power consumption is achieved.
\end{abstract}

Keywords. Network-on-Chip; latency; power; dynamic buffer allocation; RTBM router; irregular NoC.

*For correspondence 


\section{Introduction}

Reconfigurable embedded systems are Multiprocessor System-on-Chips (MPSoC) consist of Processors, DSP cores, memories, I/O blocks and IP cores. They vary in sizes and lead to the heterogeneous nature of MPSoCs. The complexity of these systems keeps growing as the number of components increases. The traditional bus based interconnection in SoC is not scalable. On-chip interconnection networks are introduced in place of ad-hoc global wiring (Dally \& Towles 2001) and led to modular design. The system modules can communicate by sending packets between each other. Hence, Networks-on-Chip (NoCs) provide scalable interconnections to connect various processor cores (Benini \& De Mircheli 2002). Parallelism can be employed in NoCs, because the simultaneous operation of channels on different data packets is possible.

In general, $\mathrm{X}-\mathrm{Y}$ routing can be used for routing in regular NoC topology. But, by the oversized IP cores of MPSoCs, NoC topology becomes irregular as shown in figure 1. As a result of the oversized IPs, some of the links may be broken. Hence, a routing algorithm that ensures communication between components in the irregular mesh topology is needed to increase reliability. Virtual-Channel based fault tolerant routing algorithms for 2D NoC (Nicopoulos et al 2006) are not adaptive with dynamically changing traffic loads. Since memory blocks are not scalable in an adaptive routing algorithm proposed in (Schonwald et al 2007), it is not suitable for large NoCs. Therefore, we apply an adaptive routing algorithm to maintain regular traffic. Using this, packets can be rerouted instantly if a link is broken. A configuration register is used in each router to maintain congestion information. Hence, there is no need of routing tables and exchange of fault

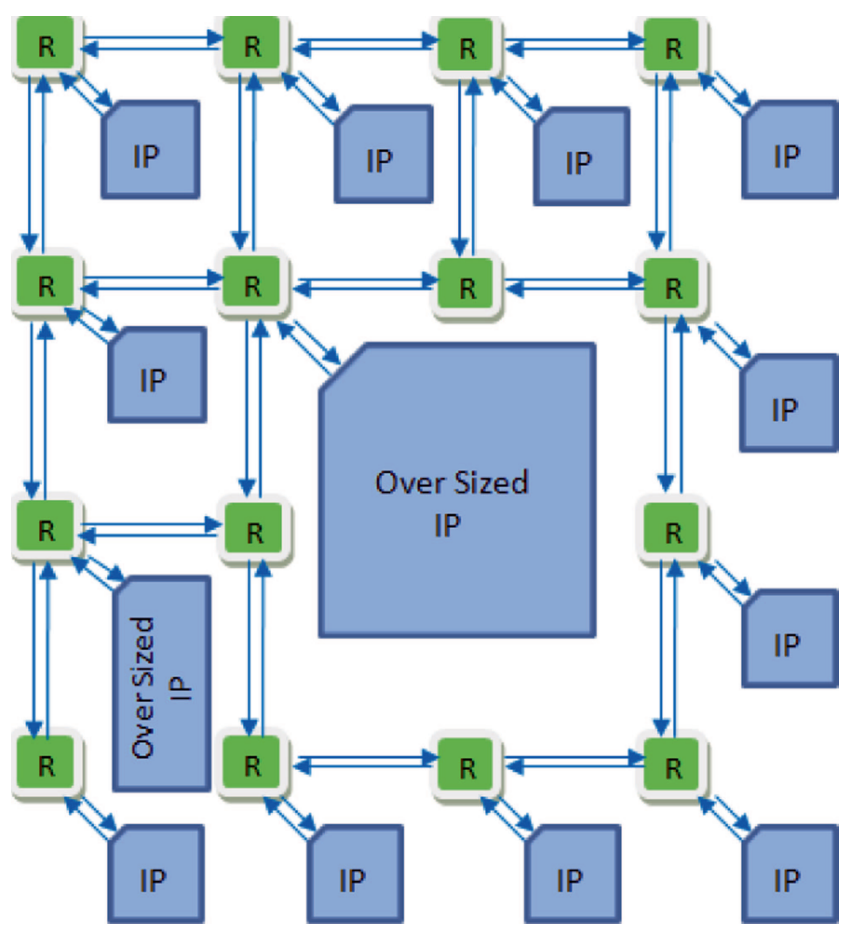

Figure 1. NoC with irregular mesh topology. 
information between routers. As the configuration registers in each router stores fault details, this algorithm can tolerate multiple broken links.

NoCs in MPSoC architecture may have different communication bandwidth under different applications. To use a single NoC router for all applications, an extremely large sized buffer which consumes more area and power is needed. On the other hand, to use a specific type of NoCs for different applications, factors such as throughput, latency, and bandwidth have to be decided at design time. Nevertheless, if the communication pattern changes dynamically, these decisions lead to poor performance or excessive consumption of area and power.

In this paper, a heterogeneous router that balances both power and latency under different communication needs is proposed. The proposed router uses small buffers and provides better performance as a large fixed size buffered router. Each input channel in the router can lend/borrow buffer slots to/from neighbors to get the required bandwidth. This paper is organized as follows. The proposed Run time buffer management algorithm for reallocation of buffer slots is explained in Section 2. In Section 3 NoC Router Architecture to support the proposed algorithms is explained. The simulation results and discussions are presented in Section 4. The detailed literature review is presented in Section 5. Also, the proposed buffer scheme is compared to buffer schemes of related work in the same section. Finally, Section 6 summarizes our findings.

\section{Runtime buffer management algorithm}

NoC router architecture consists of crossbar, FIFOs, arbiters and input/output channels or links. Large leakage power is consumed by FIFOs or buffers. A considerable amount of buffers is idle at low traffic loads in many routers. Though at some routers all the buffers are busy at high traffic load, average amount of buffers used is low. Approximately, $85 \%$ of buffers are idle on an average (Xuning \& Peh 2003). Ye et al (2002) proposed a work to analyze the power consumption on the switch fabric in NoC routers. The results of this work confirmed that power consumption by the buffer is high as its size is large.

In a NoC, the throughput can be increased and average latency can be reduced by increasing the buffer depth at the input channels of routers. But this cannot be done beyond a limit. Buffer depth designed for worst case traffic load will lead to large power consumption and silicon area (Xuning \& Peh 2003). In contrast, smaller buffer depth results in increased average latency and performance degradation.

The idle buffer slots of input channels can be efficiently utilized to improve the performance. Hotspots are those input channels in which a large number of flits arrive and all buffer slots of those channels are busy. If an input channel is less congested while its neighbor is a hotspot, then it can lend its free slots to the neighbor. The situation may reverse and the channel, which borrowed earlier, can now lend its free slots. The Runtime Buffer Management (RTBM) Algorithm shown in figure 2 manages idle buffer slots based on the number of hotspots in a router.

The algorithm, first, examines the number of hotspots in each router. If the router is in a mesh corner with one hotspot, then buffer slots from other channel are borrowed. If the router is in the mesh border and has one hotspot channel with only one neighbor, then it borrows free buffer slots from its neighbor. If it has two neighbors, it can borrow from both right and left neighbor. If there are two hotspots and both are on the same line, then free slots of the neighbor can be shared between them. Otherwise, one hotspot channel, which has not-hotspot channel as a neighbor, can borrow the free slots. But if the router is elsewhere in the mesh with one hotspot, both the neighbors can lend buffer slots. If hotspots are two, then each can borrow from one neighbor. In 


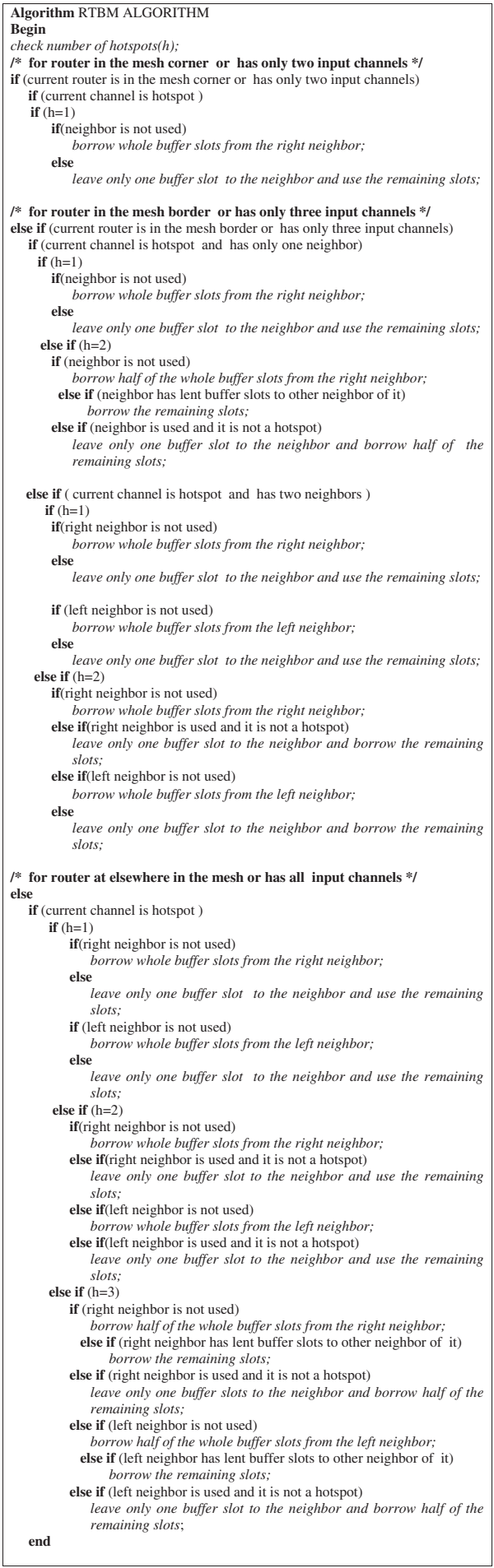

Figure 2. Runtime buffer management algorithm. 
case of three hotspots, free buffer slots of the not-hotspot channel can be shared between the two channels. When an input channel is used and not a hotspot, only one slot can be kept for its own use and other slots can be lent. This is because, when the channel is not a hotspot, the packet comes in is sent out immediately. If all the four channels are hotspots, then buffer slots cannot be lent.

\section{NoC router architecture}

We considered an irregular 2-D Mesh NoC as shown in figure 1. The modules of this architecture are developed using Verilog HDL. The proposed router used in the NoC architecture consists of five ports viz., North, East, West, South and Local port and a crossbar. The crossbar is built with five multiplexers, one for each output port. Data flits from all five input channels are given as input to the multiplexer. A round robin arbiter chooses an appropriate input channel to transmit the data when two or more channels contend to access the output channel. Hence, a router can support at the maximum of five parallel connections simultaneously.

The flow control is achieved using wormhole switching technique. By this technique, the packet is divided into smaller units called flow control units or flits and these flits are delivered in a pipelined fashion in the NoC (Zhonghai Lu 2005). Each packet has three types of flits: one header flit, one or more body flits with payload and a tail flit to say end of the packet. They are identified by two bits. The header flit of each packet has source and destination addresses. Based on this information a routing direction is decided on each node and a path will be set between source and destination. The body or payload flits will follow the path set by the header flit. The path will be terminated when the tail flit of each packet passes through a node. The benefit of the wormhole switching is that the buffer depth can be set as small as possible.

Each port has an input channel as well as an output channel. Buffers are present at all input channels to store the data temporarily at the time of congestion. There is no need of buffers at the output channel since it is enabled by a hand shaking signal from the next router. FIFO is used as the input buffer to store the flits temporarily. The width of FIFO is decided by flit size and physical buffer depth is defined at design time. The flit can be written into or read from FIFO using RTBM algorithm. Router hardware with detailed buffer logic is shown in figure 3(a) and (b). To make it simple, figure 3 presents only the eastern part of the router in detail.

The input flit to the FIFO may enter from its own input channel or from its neighbor input channels. Control logic is implemented using a Finite State Machine (FSM). When FSM receives a borrow request from its neighbor, it checks the availability of free buffer slots using FULL \& EMPTY signals. Depending upon the availability, FSM sends a borrow grant signal to the requesting neighbor and sends a select signal to the DATA IN MUX. In case, if both the neighbors are requesting and the own channel is not a hot spot, then the FIFO is partitioned into three parts. FSM uses a set of read and write pointers for own channel, right neighbor and left neighbor. If the own channel becomes hotspot, FSM sends the borrow request to the neighbors and writes its buffer words into the neighbor buffer slots if granted.

FSM saves the information about the number of the buffer slots lent to the neighbors and their positions. It also knows the number of the buffer slots used by itself and a number of its own buffer words saved in the neighbor's FIFO. When the neighbor has sent the flits from its buffer slots to the next router in the path, it wants to read its buffer word from borrowed buffer slots and sends a read request. FSM enables this read operation using the neighbor's read pointer and a select signal to DEMUX. FSM allows a flit to enter into the crossbar switch from its own buffer or its right neighbor or left neighbor by providing a select signal to DATA OUT MUX. 


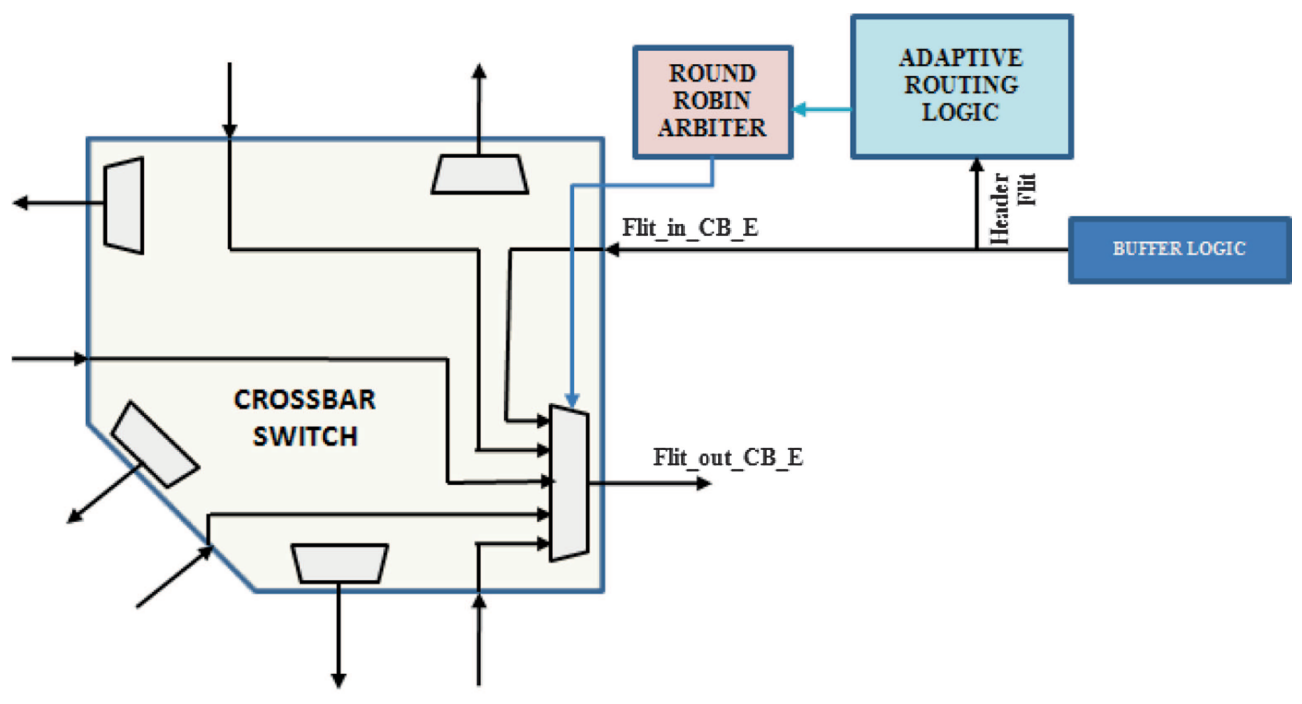

(a)

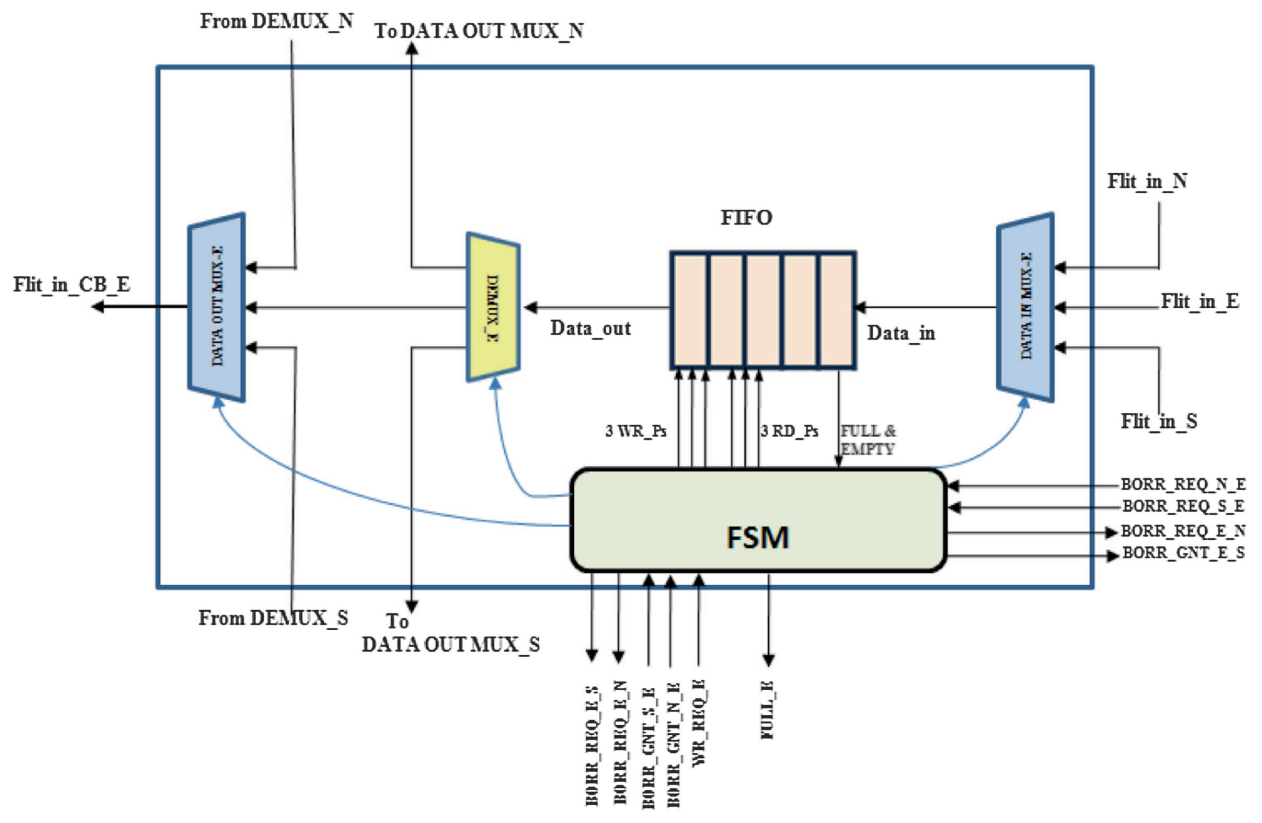

(b)

Figure 3. (a) RTBM router architecture. (b) Detailed buffer logic with FSM.

When a header flit enters into the crossbar, a copy of it is used by router logic to find the output channel for that packet. If the current router is the destination, it places a request to arbiter to select local channel. Otherwise, it places the request to select appropriate output channel. The routing logic uses a 16-bit register of neighbor broken links information to make a decision on the path. The crossbar is equipped to reroute the packet to the router where it came from when 
no path is found. This rerouting is indicated by setting one bit in the header flit. By checking this bit, the router changes the route of the packet and thus avoids cycling of the packet.

\section{Results and discussions}

For all our experiments, irregular two dimensional mesh topologies with adaptive routing algorithm are considered. The performance of the proposed RTBM router is compared with that of a static buffer router and the effectiveness of RTBM router is evaluated. We implemented two $4 \times 4$ irregular mesh NoCs, one with proposed router and the other with the static buffer router. Simulations are carried out by randomly choosing source-destination pairs and results are evaluated.

To increase the complexity in evaluation, $8 \times 8$ irregular mesh NoCs are considered. The simulation results are obtained using the synthetic traffic patterns, namely, uniform, bit complement, tornado and hotspot traffics. In uniform traffic, any node can be the source and any other can be the destination with random selection. If $(x, y)$ is the source node, then the destination node is $((x+3) \% 8,(y+3) \% 8)$ in tornado traffic and $(\sim x, \sim y)$ in bit complement traffic. In hotspot traffic, some nodes are fixed as hotspots. Twenty percent of the traffic is routed towards them and the remaining $80 \%$ is uniform.

Further, we used Embedded System Synthesis Benchmarks Suite (Dick E3S) which is based on the data from the Embedded Microprocessor Benchmark Consortium (EEMBC) to evaluate and compare the performances of both the routers for realistic traffic patterns. E3S benchmark suite consists of five applications: auto/indust, networking, telecom, consumer and officeautomation. The task graphs of these applications are mapped into irregular mesh NoCs of required size and the simulation experiments are carried out using a cycle accurate simulator. The results of all the experiments and evaluations are presented in the following sections.

\subsection{Latency and throughput results in $4 \times 4$ irregular mesh $\mathrm{NoC}$}

The performances of a $4 \times 4$ irregular mesh $\mathrm{NoC}$, as shown in figure 1 , with the static buffer router and with the proposed router architecture are evaluated using a cycle accurate RTL simulator. We assumed four traffic scenarios, each with different source-destination pairs. One scenario, for example, is shown in figure 4. Source nodes and destination nodes are identified by the letters $S$ and $D$ respectively, with a packet number as a suffix. The paths of the packet are shown in different colored arrows. In other scenarios, different combinations of source-destination pair are considered.

The simulations are carried out for every scenario with five packets injected from the source nodes. Each packet has a fixed length of 128 flits and a flit width of 32 bits. A packet generator is implemented in Verilog HDL and attached with every router. The number of cycles required to deliver each packet, i.e. number of cycles between injection of header flit from the source and acceptance of tail flit at destination generally called the latency of the packet, is measured and tabulated in tables $1-4$.

Congestion in a channel occurs when more than one packet shares the channel. The results presented in tables 1-4 show that latency of a packet is reduced by RTBM router during congestion. If there is no congestion in the path, then latency is the same irrespective of RTBM router used or not.

Traffic pattern and traffic rate will be different from application to application. Hence, packet injection rate and in turn flit injection rate (flits/cycle/IP) in the NoC vary with time. As the flit 


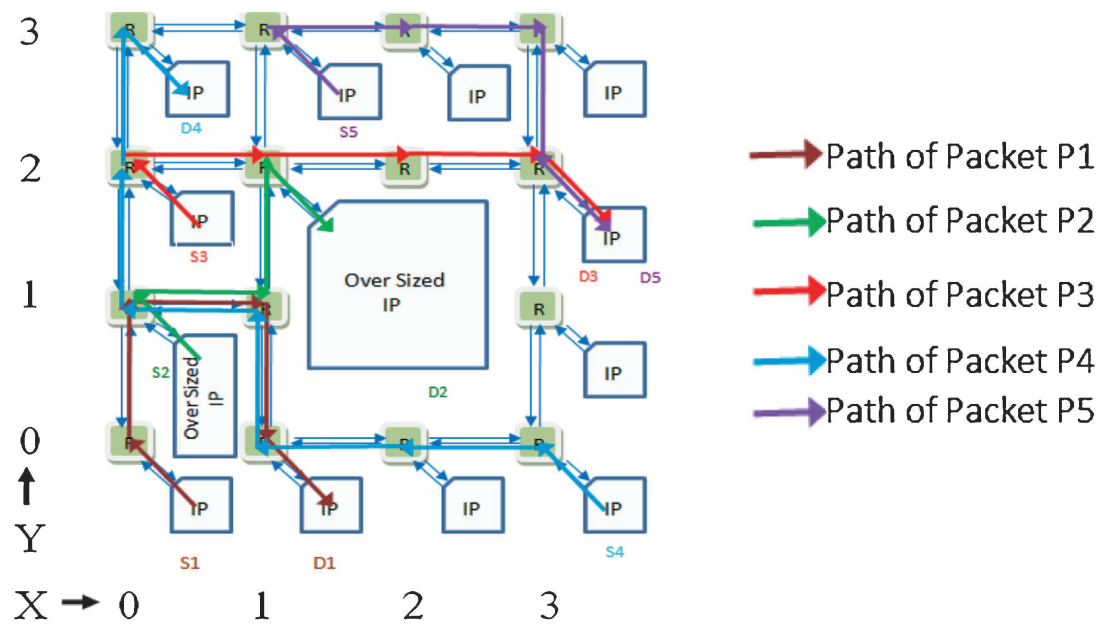

Figure 4. Traffic scenario 1.

Table 1. Measurement of Latency of each packet in scenario 1.

\begin{tabular}{lccc}
\hline Packet number & $\begin{array}{c}\text { Source- } \\
\text { destination } \\
\text { pair }\end{array}$ & $\begin{array}{c}\text { Latency } \\
\text { (cycles) with } \\
\text { static router }\end{array}$ & $\begin{array}{c}\text { Latency } \\
\text { (cycles) with } \\
\text { RTBM router }\end{array}$ \\
\hline P1 & S1 $(0,0)-D 1(1,0)$ & 264 & 256 \\
P2 & S2 $(0,1)-$ D2(1,2) & 132 & 132 \\
P3 & S3 (0,2)-D3(3,2) & 268 & 250 \\
P4 & S4(3,0)-D4(0,3) & 134 & 134 \\
P5 & S5 (1,3)-D5(3,2) & 138 & 138 \\
\hline
\end{tabular}

Table 2. Measurement of Latency of each packet in scenario 2.

\begin{tabular}{lccc}
\hline Packet number & $\begin{array}{c}\text { Source- } \\
\text { destination } \\
\text { pair }\end{array}$ & $\begin{array}{c}\text { Latency } \\
\text { (cycles) with } \\
\text { static router }\end{array}$ & $\begin{array}{c}\text { Latency } \\
\text { (cycles) with } \\
\text { RTBM router }\end{array}$ \\
\hline P1 & S1(0,1)-D1(2,0) & 640 & 632 \\
P2 & S2(1,0)-D2(3,0) & 514 & 502 \\
P3 & S3(2,0)-D3(3,1) & 388 & 378 \\
P4 & S4(1,2)-D4(3,2) & 134 & 134 \\
P5 & S5(3,0)-D5(3,2) & 262 & 254 \\
\hline
\end{tabular}

Table 3. Measurement of Latency of each packet in scenario 3.

\begin{tabular}{lccc}
\hline Packet number & $\begin{array}{c}\text { Source- } \\
\text { destination } \\
\text { pair }\end{array}$ & $\begin{array}{c}\text { Latency } \\
\text { (cycles) with } \\
\text { static router }\end{array}$ & $\begin{array}{c}\text { Latency } \\
\text { (cycles) with } \\
\text { RTBM router }\end{array}$ \\
\hline P1 & S1(3.3) D1(0,0) & 253 & 241 \\
P2 & S2(0,3) D2(2,0) & 137 & 137 \\
P3 & S3(3,0) D3(1,2) & 134 & 134 \\
P4 & S4(3,1) D4(1,1) & 385 & 373 \\
P5 & S5(0,2) D5(0,1) & 130 & 130 \\
\hline
\end{tabular}


Table 4. Measurement of Latency of each packet in scenario 4.

\begin{tabular}{lccc}
\hline Packet number & $\begin{array}{c}\text { Source- } \\
\text { destination } \\
\text { pair }\end{array}$ & $\begin{array}{c}\text { Latency } \\
\text { (cycles) with } \\
\text { static router }\end{array}$ & $\begin{array}{c}\text { Latency } \\
\text { (cycles) with } \\
\text { RTBM router }\end{array}$ \\
\hline P1 & S1(2,0) D1(0,1) & 133 & 133 \\
P2 & S2(3,0) D2(1,0) & 262 & 254 \\
P3 & S3(3,1) D3(2,0) & 388 & 378 \\
P4 & S4(3,2) D4(3,0) & 514 & 502 \\
P5 & S5(0,2) D5(1,2) & 131 & 131 \\
\hline
\end{tabular}

injection rate increases, congestion in the NoC channels increases. Therefore, we examined the performance of NoC for varying flit injection rate. Figure 5 demonstrates how the performance measures, like average latency, throughput and no. of flits dropped, vary with increasing flit injection rate in NoCs with RTBM Router and Static Router.

The average latency is calculated using Eq. (1).

$$
L_{\text {avg }}=\frac{\sum_{i=1}^{P} L_{i}}{P}
$$

where $P$ is the number of packets received in a given time period and $L i$ is the latency of the $i$ th the packet.

Figure 5(a) illustrates that there is an improvement in latency with dynamic buffer allocation using RTBM compared with static buffer allocation. Throughput is also measured and compared for static and dynamic buffer allocation schemes. Figure 5(b) shows that throughput linearly increases for lower injection rates and is same for both the schemes. As injection rate increases further, congestion increases and more flits are dropped when static buffer allocation used, leads to fall in throughput compared to the dynamic buffer allocation.

The number of cycles that the flits have to wait at source nodes for buffer availability to get injected into the NoC is determined for increasing the injection rate and depicted in figure 5(c). With the use of RTBM router the unused neighbor buffers are utilized during congestion, the wait cycles are lesser when compared static router. This shows that runtime buffer allocation results in efficient buffer utilization and hence power reduction.

\subsection{Buffer requirement and resource utilization}

To achieve the same amount of average latency with RTBM, the buffer depth is increased in the case of the static buffer allocation scheme. Figure 6 depicts the total buffer slots required in the irregular mesh NoCs with RTBM router and static router. It shows that the buffer size increases with flit injection rate when RTBM router is not used. For the applications with high injection rate, the buffer requirement is almost halved with the help of RTBM. Hence, the area requirement for buffers and the power consumption are considerably reduced.

The heterogeneous router with RTBM and the static router are synthesized using an EDA tool and realized in an FPGA. The hardware requirement is compared in figure 7 . It shows that the area overhead of the heterogeneous router is $27.51 \%$ in LUTs and $15.17 \%$ in FFs. But this area overhead is compensated by smaller buffer size requirement. 


\section{Average Latency Vs Flit Injection Rate}

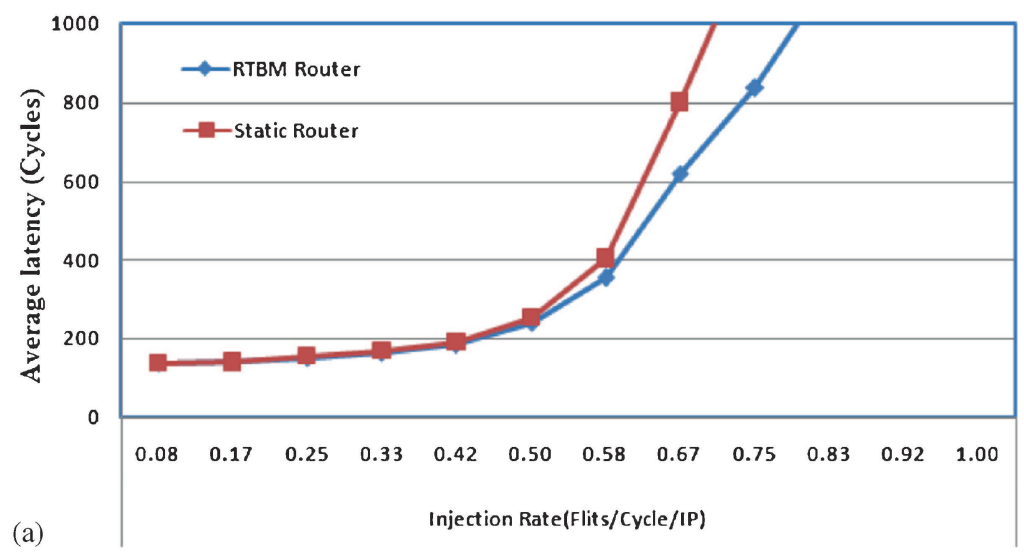

Throuhput Vs Flit Injection Rate

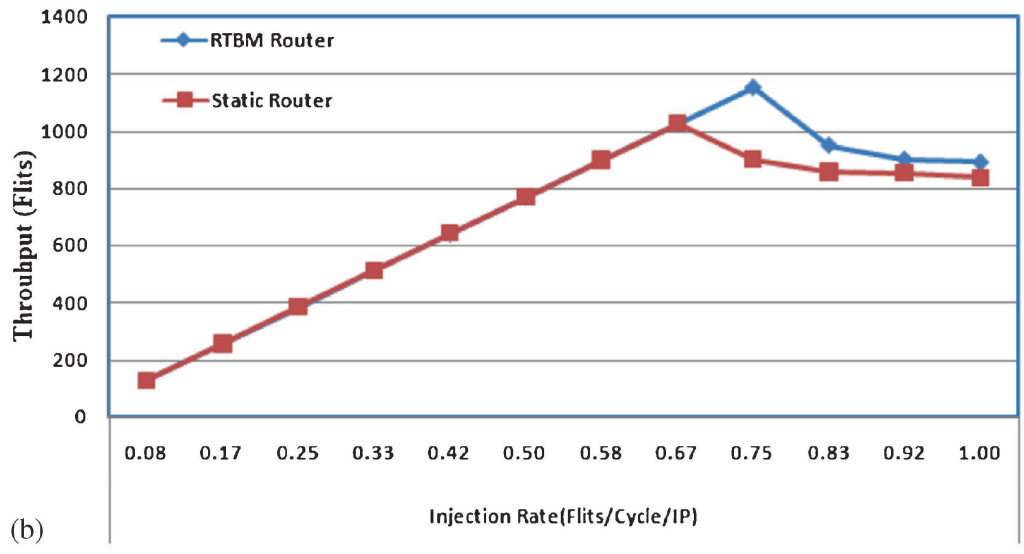

No. of waiting cycles Vs Flit Injection Rate

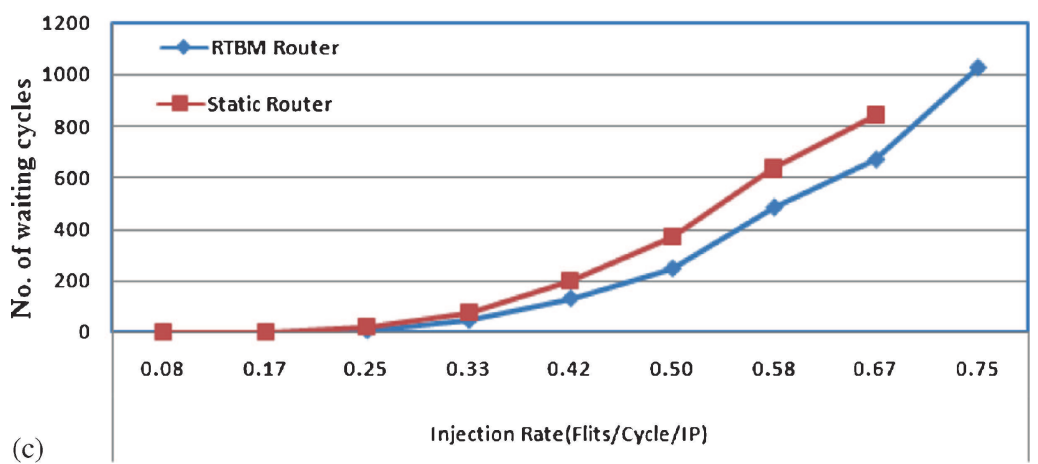

Figure 5. Performance measures with a packet size of 128 flits of 32-bits each. (a) average latency, (b) throughput, and (c) no. of wait cycles at source nodes. 


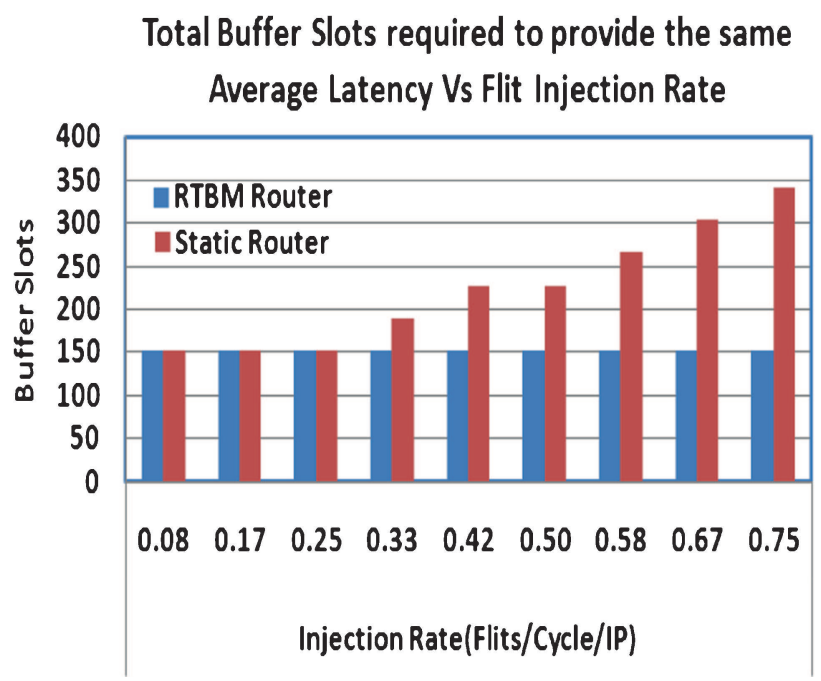

Figure 6. Buffer slots required for the same average latency.

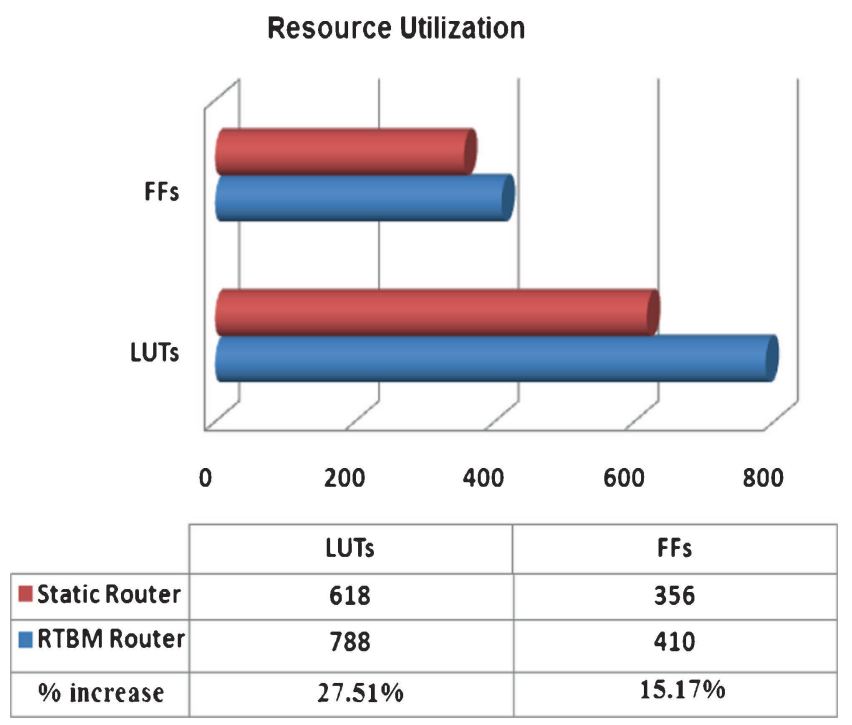

Figure 7. Comparison with respect to router resource utilization.

\subsection{Latency and throughput results in $8 \times 8$ irregular mesh NoC}

An $8 \times 8$ irregular mesh NoC with RTMB router is constructed by replicating the $4 \times 4$ NoCs and is shown in figure 8. Packets of four 32-bit flits are injected into the NoC using synthetic traffics like uniform, bit complement, tornado and hotspot traffics.

In each case, the average latency is measured for increasing injection rates. The same experiments are carried out in $8 \times 8$ irregular mesh NoC with two variants of static router; Buffer depth of 4 and buffer depth of 10. In figure 9, the average latency of packets is plotted against the increasing injection rate for the synthetic traffic patterns. 


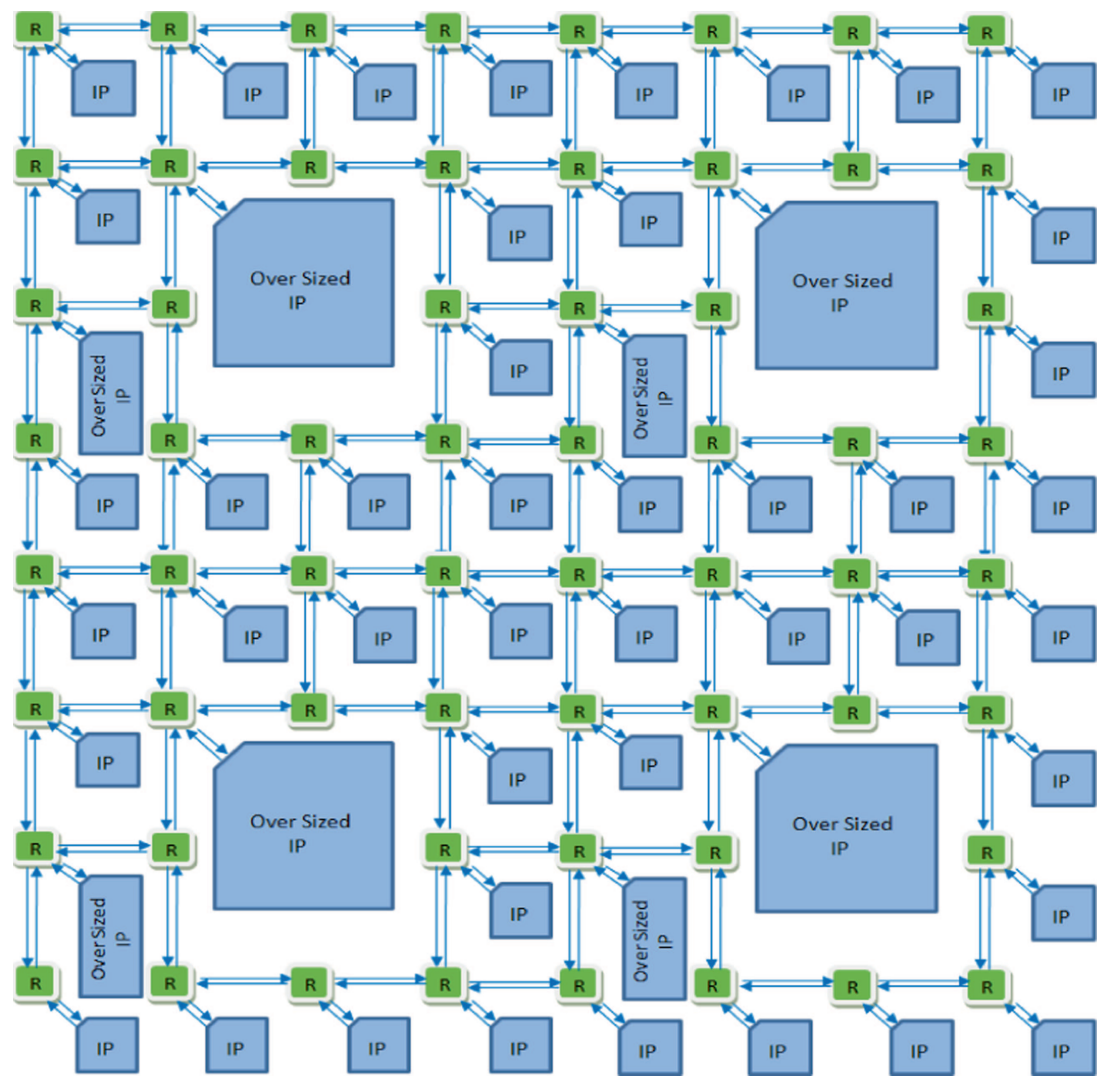

Figure 8. An $8 \times 8$ irregular mesh NoC.

Normalized saturation throughput is measured as the injection rate at which the average latency increases sharply and shown in figure 10. Latency and throughput are improved by the proposed router for all the four synthetic traces compared to static router with buffer depth of 4 . They are approximately the same as that of static router buffer depth of 10 and for this reason we considered a static router and RTBM router, both with the buffer depth of 4 to compare the performance measures.

The average latency improvement in NoC with RTBM router compared to the static router buffer depth of 4 is $22.08 \%, 35.68 \%, 19.02 \%$ and $46.1 \%$ for uniform, bit complement, tornado and hotspot traffics respectively. In uniform traffic, the packets are sent to randomly chosen nodes. Therefore, the performance improvement is nominal with a throughput increase of $8.9 \%$. The average latency reduction is almost double in case of bit complement traffic compared to tornado traffic. Source-destination pairs are relatively closer in tornado traffic. Therefore, the need of dynamic buffer allocation is less and hence it results in lesser latency improvement. But, NoC saturates at a $20 \%$ earlier injection rate in bit complement traffic as a result of additional congestion due to long hop distances.

The proposed router outperforms in hotspot traffic with respect to the other synthetic traffics. This proves that the proposed router handles hotspots efficiently compared to static buffer router and reduces congestion in NoC. Although the saturation throughput is the least for hotspot traffic, the improvement in throughput achieved by the RTBM router over static router is the highest 

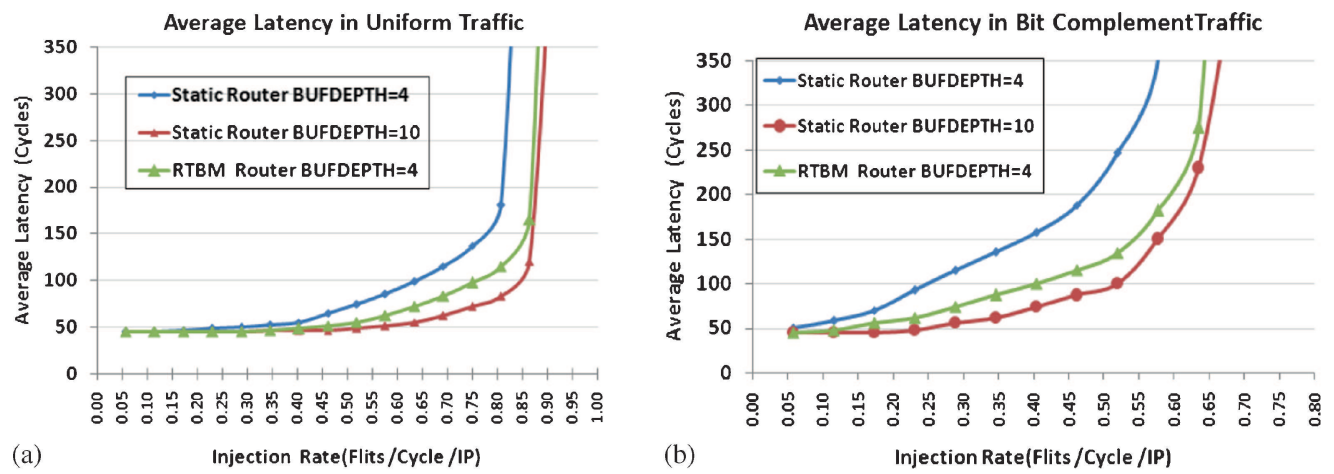

(b)

Injection Rate(Flits/Cycle /IP)
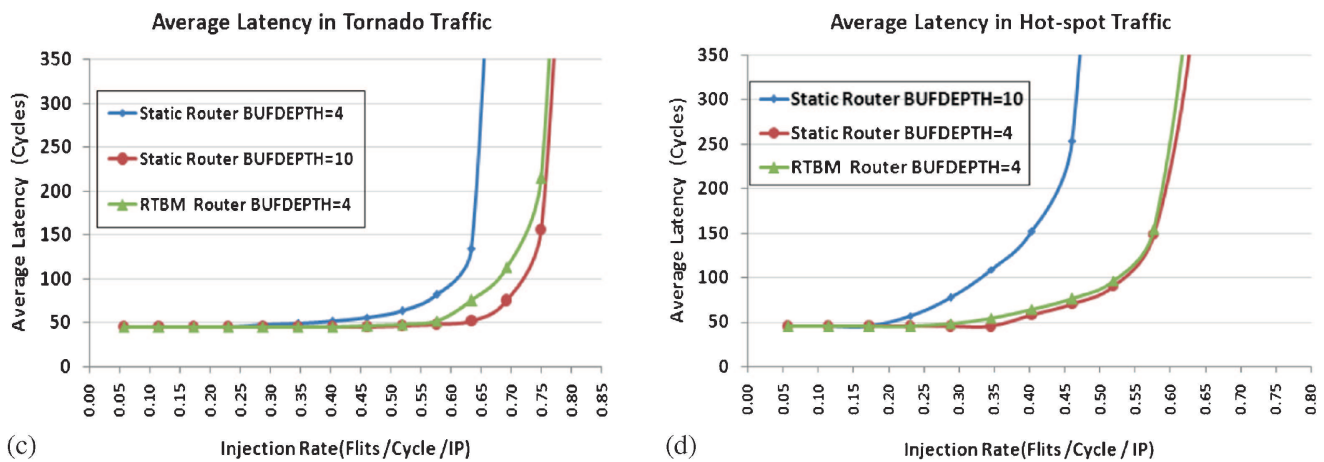

Figure 9. Performance comparison of NoCs with RTBM router and Static router for the synthetic traffics (a) Uniform, (b) Bit complement, (c) Tornado and (d) Hotspot.

among all the four synthetic traffics. Throughput improvement in uniform, bit complement, tornado and hotspot traffics are $8.9 \%, 12.28 \%, 18.75 \%$ and $33.33 \%$ respectively. The overall average latency improvement of $30.42 \%$ and overall average saturation throughput improvement of $18.33 \%$ are achieved by the proposed router for the four synthetic traffics.

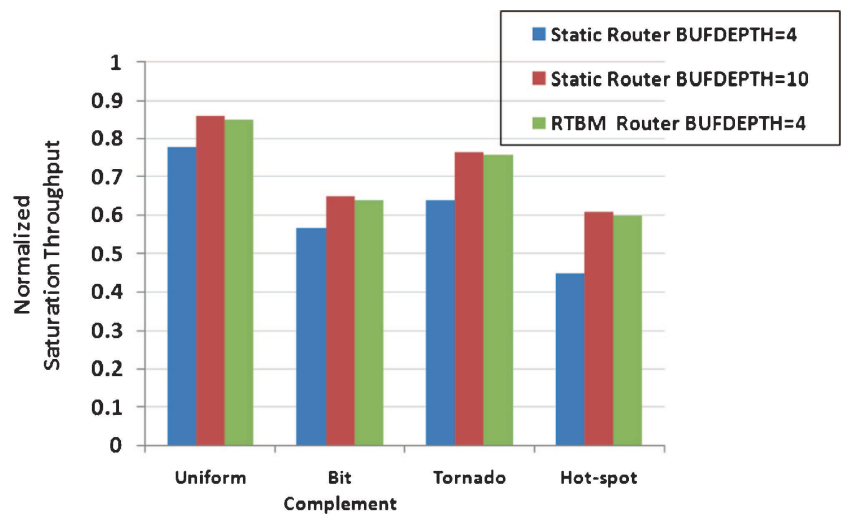

Synthetic Traffic Type

Figure 10. Normalized Throughput for synthetic traffic patterns. 


\subsection{Latency and throughput results for E3S benchmark suite}

We used E3S benchmark suite to demonstrate the performance enhancement of the proposed RTBM router compared to the static router for the realistic traffic patterns. There are five applications, namely, automotive/industrial, networking, consumer, telecommunications, and office automation in this benchmark suite. The task graphs of these applications consist of $24,13,12$, 30 and 5 tasks respectively. Correspondingly, these task graphs are mapped into $5 \times 6,4 \times 4,6$ $\times 6,4 \times 4$ and $3 \times 3$ irregular mesh NoCs. Traffic is generated as per the communication volumes between the tasks mentioned in the benchmark for each application. For each application, simulation is carried out for a sufficient number of cycles in order to run the complete application and this number varies for each application.

The latencies of packets are observed and the average of packet latencies is computed for each application in NoC with RTBM router buffer depth of 4 . The similar procedure is followed in $\mathrm{NoC}$ with the static router buffer depth of 4 . The average latency in NoC for both the routers is presented in figure 11. It can be observed that the average packet latency reduced by the proposed router for all the five applications. In office automation application, reduction in latency is very less, because the size of the NoC is very small and reduction in latency by RTBM router is also insignificant.

Although the size of the NoCs for the networking and consumer applications is same, the average latency for consumer application is more than that of networking application. This is because the communication volume in consumer application is almost double due to image processing tasks like color filters, compression and decompression. The latency improvement attained by the proposed router in consumer application is slightly greater than the latency improvement achieved in the networking application.

The average packet latency is larger for the telecom and automotive/industrial applications, in contrast to the other applications. The reason is that the number of tasks involved in these two applications. Among them, the telecom application suffers from higher latency compared to the auto/indust application. Both the applications involve tasks like fft, auto correlation, and convolution encoder. The enhancement achieved in latency by the RTBM router is better for telecom

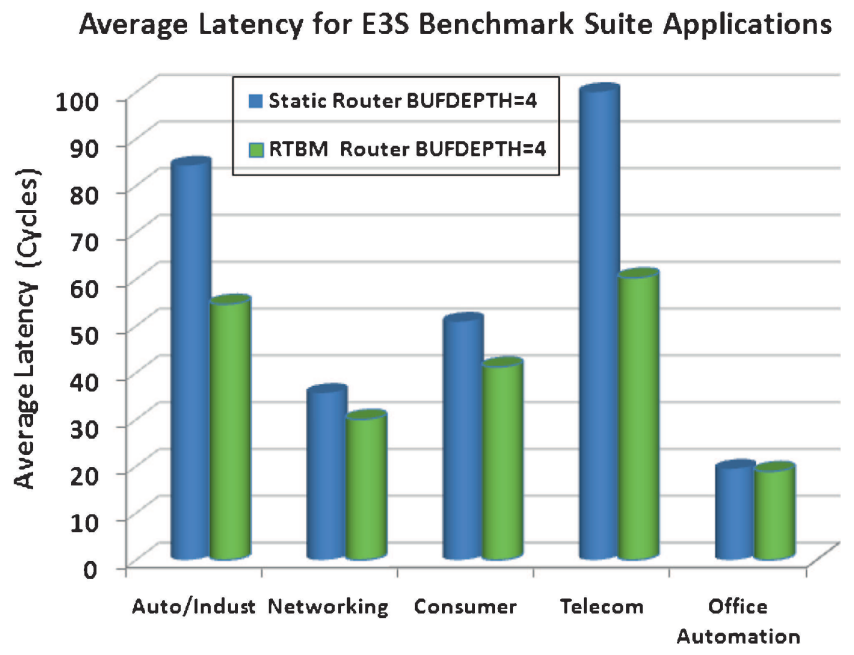

Figure 11. Latency in NoCs for E3S benchmark applications. 
application than all other applications. The percentage reduction in average latency for automotive/industrial, networking, consumer, telecommunications, and office automation applications are $35.17 \%, 15.84 \%, 19.03 \%, 40.23 \%$ and $2.8 \%$ respectively. Therefore, the overall latency reduction for the E3S benchmark suite is $22.63 \%$ by the proposed dynamic RTBM router.

\subsection{Power and area results}

The RTBM router and static router are described in Verilog HDL. The average power consumption and area are estimated using Cadence Encounter(R) RTL Compiler with CMOS $180 \mathrm{~nm}$ standard cell library. The design operates at a supply voltage of $1.8 \mathrm{~V}$, and the power results were obtained with a $1 \mathrm{GHz}$ clock frequency in both the router architectures with the FIFO buffer depth as 4 . The power and area results obtained are presented in table 5. The power and area are measured for the static router with the buffer depth of 10 and tabulated.

Table 5. Power and area results of RTBM router architecture and static route architecture.

\begin{tabular}{|c|c|c|c|c|c|c|c|c|c|c|c|c|}
\hline \multirow[b]{2}{*}{$\begin{array}{l}\text { Router } \\
\text { architecture }\end{array}$} & \multicolumn{6}{|c|}{ Power(mW) } & \multicolumn{6}{|c|}{ Area $\left(\mathrm{mm}^{2}\right)$} \\
\hline & Arbiter & $\begin{array}{c}\text { Adaptive } \\
\text { routing } \\
\text { logic }\end{array}$ & $\begin{array}{c}\text { Crossbar } \\
\text { switch }\end{array}$ & FIFO & FSM & $\begin{array}{c}\text { Total } \\
\text { power }\end{array}$ & Arbiter & $\begin{array}{c}\text { Adaptive } \\
\text { routing } \\
\text { logic }\end{array}$ & $\begin{array}{c}\text { Crossbar } \\
\text { switch }\end{array}$ & FIFO & FSM & $\begin{array}{l}\text { Total } \\
\text { area }\end{array}$ \\
\hline $\begin{array}{l}\text { Static } \\
\text { router } \\
\text { (buffer } \\
\text { depth =4) }\end{array}$ & 2.4 & 1.1 & 2.7 & 40.6 & - & 46.8 & 0.005 & 0.003 & 0.02 & 0.075 & - & 0.103 \\
\hline $\begin{array}{l}\text { RTBM } \\
\text { router } \\
\text { (buffer } \\
\text { depth =4) }\end{array}$ & 2.4 & 1.1 & 2.7 & 40.6 & 16.9 & 63.7 & 0.005 & 0.003 & 0.02 & 0.075 & 0.064 & 0.167 \\
\hline $\begin{array}{l}\text { Static } \\
\text { router } \\
\text { (buffer } \\
\text { depth = 10) }\end{array}$ & 2.4 & 1.1 & 2.7 & 91.3 & - & 97.5 & 0.005 & 0.003 & 0.02 & 0.3 & - & 0.328 \\
\hline
\end{tabular}

Table 6. Comparison of latency, throughput, power and area (normalized with respect to static router buffer depth of 4).

\begin{tabular}{lcccc}
\hline $\begin{array}{l}\text { Parameter } \\
\text { (normalized) }\end{array}$ & $\begin{array}{c}\text { Traffic } \\
\text { pattern }\end{array}$ & $\begin{array}{c}\text { Static router } \\
\text { (buffer depth =4) }\end{array}$ & $\begin{array}{c}\text { RTBM router } \\
\text { (buffer depth }=4)\end{array}$ & $\begin{array}{c}\text { Static router } \\
\text { (buffer depth = 10) }\end{array}$ \\
\hline Average latency & $\begin{array}{c}\text { Uniform } \\
\text { Bit complement } \\
\text { Tornado }\end{array}$ & 1 & 0.78 & 0.72 \\
& Hotspot & 1 & 0.65 & 0.59 \\
Saturation throughput & Uniform & 1 & 0.81 & 0.79 \\
& Bit complement & 1 & 0.54 & 0.51 \\
& Tornado & 1 & 1.09 & 1.10 \\
Power & Hotspot & 1 & 1.12 & 1.14 \\
Area & - & 1 & 1.19 & 1.20 \\
& - & 1 & 1.33 & 2.08 \\
\hline
\end{tabular}


The comparison of parameters in both the RTBM router and the static router with buffer depths of 4 and 10 is shown in table 6 . All these parameters are normalized with respect to static router buffer depth of 4 . Average latency and saturation throughput presented for four types of synthetic traffics. With the buffer depth of 4 , the power penalty in RTBM router is $36 \%$, but the overall average latency is reduced by $30.42 \%$ and saturation throughput is improved by $18.33 \%$ when compared to the static router buffer depth of 4 . It can be observed that approximately the same performance is achieved in all traffic patterns by the RTBM router and the static router buffer depth of 10. But the power reduction achieved with RTBM router compared to the static router buffer depth of 10 is $53 \%$. The area penalty is only 1.62 times in RTBM router compared to 3.18 times in static router buffer depth of 10 to provide the more or less same latency and throughput.

\section{Related work}

To tolerate broken links and routers, a routing method that supports irregular topologies in mesh-based NoCs is proposed by (Valinataj \& Mohammadi 2010). It concurrently uses both fault and congestion information to route the packets. Significant reliability improvement is achieved against multiple component failures. It is suitable for NoC applications in unreliable environments. In the heterogeneous router (Matos et al 2011), each channel can have a different buffer size and the buffer slots are allocated dynamically to improve the performance. Only two hotspots are considered among the four channels. $X-Y$ routing algorithm is used considering regular 2D mesh Topology. It does not support irregular topology. A buffer stealing method was proposed by (Su et al 2011). The input channels which do not have enough buffer slots steal the unused buffer slots from neighboring channels. However, the thief buffers and victim buffers should be fixed at design time. So, if victim buffer does not have enough space, it cannot steal from its neighbors. Different clocks are used for sending a head flit and body flits in NoC (Lee \& Bager Zadeh 2006). Body flits follow head flit and do not need any computation to find the next router in the path. Hence, they can be sent at a faster clock rate. The clock boosting mechanism proposed in this paper increases the throughput of the original adaptive router by increasing the accepted load and decreases the average latency.

The authors presented a proposal using a bus like interface in the network router (Ahmad et al 2008). A built-in-wrapper is used to integrate any component compatible with a bus, into the NoC architecture. It reduces the design time and makes integration easy, since there is no need to know about the NoC architecture. A hierarchical NoC can cope with inefficiencies in a regular NoC (Ahonen \& Nurmi 2007). Here, two types of on-chip networks are used. (i) Global Network (NoC); (ii) Local Network (shared bus) which is used to connect slaves to a master that together are called local clusters. Noise is used to connect all local clusters with similar functional behavior. The works presented above (Lee \& Bager Zadeh 2006; Ahmad et al 2008; Ahonen \& Nurmi 2007) are developed to be used at design time with a static approach. Scalability problem occurs whenever NoC is used for a new application in the same platform. A buffer allocation method is used at the system level for each specific application (Jingcao et al 2006). The algorithm, making buffer distribution, is based on the architectural parameters. The parameters are modeled in $\mathrm{C}++$ and the algorithm gives a certain number of buffers for each channel. However, buffer sizing is developed at design-time for each target application, and then, if the communication behavior changes, probably the system will not deliver the required performance or the resources will be under-utilized.

A dual crossbar design is proposed by (Yixuan Zhang et al 2011) to improve the performance and reduce the power. In this system, they designed a primary crossbar with no buffers and 
a secondary cross bar with buffers. It takes the benefit of power-efficient bufferless NoCs for low traffic rates, and cost-efficient congestion managing capability at high traffic rates. But, the area overhead is doubled when compared with buffered schemes. Al Faruque \& Henkel (2008) proposed a work to optimize the number of Virtual Channels in two steps. The first step maps the tasks to minimize the virtual channels, and the second step uses an analytical approach to further optimize the number of virtual channels buffers (VCBs). The results of this paper show that there is a decrease in the number of VCBs when compared with the QNoC architecture, but no performance and/or power results were given.

An adaptive architecture with runtime observability, ROAdNoC, is proposed by (Al Faruque et al 2008), providing adaptability at the system level and at the architecture level. At system level, it can remap the tasks of the system, and at the architecture level, it can reroute the packets and reallocate the virtual channels buffers (VCB). The changes at the architecture level depends on the occurrence of a fault, and occur when the packets are not able to reach the destination or when the VCB is full. The adaptive process takes place if a fault is found, and hence no performance or power advantage can be obtained during the normal condition of the system. Nicopoulos et al (2006) proposed a work with a goal similar to the proposed work is done, but using virtual channels. A unified buffer structure with a dynamic virtual channel regulator, called ViChaR, which dynamically allocates virtual channels and buffers according to network traffic conditions, is proposed. In this case, instead of individual and statically partitioned buffers, they utilized a unified buffer. The ViChaR has many memory units to allow the correct functioning of the virtual channels. In addition to these buffers, ViChaR uses table control logic and additional registers that consume much power.

The comparison of the proposed method and few related works is presented in table 7 . The input virtual channel buffers are replaced by Elastic Buffer (Michelogiannakis et al 2009) and hence VCs are avoided. For deadlock avoidance the physical channels are duplicated. EBs are power and area efficient compared to VCs. As there are no buffers used in Elastic buffer router, performance is limited compared to $\mathrm{VC}$ router. To address this limitation, the authors proposed Hybrid EB-VC router (Michelogiannakis \& Dally 2013). A hybrid EB-VC router supports different traffic classes. Hybrid routers operate as EB routers at a low traffic rate and input buffers in VCs are used to drain flits during contention or deadlock. In EB networks with flattened butterfly topology (FBFly), universal globally adaptive load-balancing (Singh 2005) routing algorithm is used and in mesh topology dimension order routing is used.

Tri-state repeaters in the links function as buffers (Di Tomaso et al 2012) with a control logic. Number of repeater stages per virtual channel is varied and the performances are compared. Use of virtual channels avoids HoL blocking. For area efficiency, CTorus topology is used in this work and compared with other regular topologies, Mesh, CMesh and FBFly. The parameters are defined at design time and hence every application needs power, area and performance trade-offs.

Distributed Shared Buffer (DSB) router (Ramanujam et al 2011) uses buffers referred as middle memories between two stages of crossbars. The performance of noise is enhanced while comparing to input buffer scheme with a compromise on power and area overhead. A hardware regulator (Jafari et al 2010) is used between source IP and network interface to regulate traffic. The parameters of this regulator and buffer requirements are optimized using multiobjective optimization method; buffer size and buffer variance. This scheme considerably reduces the buffer requirements. The optimization is application specific and hence adaptability for different applications is limited. Flexible router (Sayed et al 2012) allows to share the input channel buffers by the packets and hence avoids VCs. But this NoC suffers from out of order reception of packets at destinations. 
Table 7. Comparison of proposed work with related work.

\begin{tabular}{|c|c|c|c|c|c|}
\hline Buffer plan & $\begin{array}{l}\text { Use of virtual } \\
\text { channels }\end{array}$ & Topology & $\begin{array}{l}\text { Routing } \\
\text { algorithm }\end{array}$ & Advantages & Limitations \\
\hline $\begin{array}{l}\text { Elastic Buffer } \\
\text { (Michelogiannakis et al } \\
\text { 2009) }\end{array}$ & $\begin{array}{l}\text { Physical channels } \\
\text { are duplicated to } \\
\text { handle different } \\
\text { traffic classes }\end{array}$ & $\begin{array}{l}\text { 2D Mesh } \\
\text { 2D FBFly }\end{array}$ & $\begin{array}{c}\text { DOR } \\
\text { UGAL } \\
(\text { Singh 2005) }\end{array}$ & $\begin{array}{c}\text { No HoL } \\
\text { blocking, } \\
\text { Power and } \\
\text { Area } \\
\text { Efficient }\end{array}$ & $\begin{array}{c}\text { Limited } \\
\text { performance }\end{array}$ \\
\hline $\begin{array}{l}\text { Hybrid } \\
\text { EB-VC } \\
\text { (Michelogiannakis \& } \\
\text { Dally 2013) }\end{array}$ & $\begin{array}{l}\text { Virtual channels } \\
\text { are used and no of VCs } \\
\text { are fixed at design } \\
\text { time }\end{array}$ & $\begin{array}{l}\text { 2D Mesh } \\
\text { 2D FBFly }\end{array}$ & $\begin{array}{c}\text { DOR } \\
\text { UGAL } \\
(\text { Singh 2005) }\end{array}$ & $\begin{array}{c}\text { No HoL } \\
\text { blocking, } \\
\text { Performance } \\
\text { and Power } \\
\text { efficient }\end{array}$ & $\begin{array}{c}\text { Area } \\
\text { overhead }\end{array}$ \\
\hline $\begin{array}{l}\text { Tri-state Link } \\
\text { Repeaters as } \\
\text { Buffers } \\
\text { (Di Tomaso et al 2012) }\end{array}$ & $\begin{array}{l}\text { Virtual channels are } \\
\text { fixed at design time }\end{array}$ & $\begin{array}{l}\text { 2D Mesh } \\
\text { 2D FBFly } \\
\text { CMesh } \\
\text { CTorus }\end{array}$ & DOR & $\begin{array}{l}\text { No HoL } \\
\text { blocking, } \\
\text { power and } \\
\text { area } \\
\text { efficient }\end{array}$ & $\begin{array}{l}\text { Each } \\
\text { application } \\
\text { requires } \\
\text { different } \\
\text { trade-offs }\end{array}$ \\
\hline $\begin{array}{l}\text { Distributed Shared } \\
\text { Buffer, DSB } \\
\text { (Ramanujam et al 2011) }\end{array}$ & $\begin{array}{l}\text { Virtual channels are } \\
\text { fixed at design time }\end{array}$ & 2D Mesh & DOR-XY & $\begin{array}{c}\text { Performance } \\
\text { enhanced }\end{array}$ & $\begin{array}{l}\text { Power and } \\
\text { area } \\
\text { overhead }\end{array}$ \\
\hline $\begin{array}{l}\text { Optimized } \\
\text { regulator with } \\
\text { buffers } \\
\text { (Jafari et al 2010) }\end{array}$ & $\begin{array}{c}\text { Number of virtual } \\
\text { channels are fixed at } \\
\text { design time }\end{array}$ & 2D Meshs & DOR-XY & $\begin{array}{l}\text { IPs are not } \\
\text { stalled due to } \\
\text { congestion; } \\
\text { power and } \\
\text { area } \\
\text { efficient }\end{array}$ & $\begin{array}{c}\text { Design is } \\
\text { application } \\
\text { dependent; No } \\
\text { adaptability } \\
\text { for different } \\
\text { application }\end{array}$ \\
\hline $\begin{array}{l}\text { Flexible } \\
\text { router } \\
\text { (Sayed et al 2012) }\end{array}$ & $\begin{array}{l}\text { No virtual channels } \\
\text { used; run time } \\
\text { adaptability }\end{array}$ & 2D Mesh & DOR-XY & $\begin{array}{l}\text { Power, area } \\
\text { efficient; } \\
\text { adaptable } \\
\text { for different } \\
\text { applications }\end{array}$ & $\begin{array}{c}\text { Out of order } \\
\text { reception of } \\
\text { packets; no } \\
\text { HoL } \\
\text { blocking } \\
\text { avoidance }\end{array}$ \\
\hline RTBM (proposed) & $\begin{array}{l}\text { No virtual channels } \\
\text { used; run time } \\
\text { adaptability for } \\
\text { buffer depth }\end{array}$ & $\begin{array}{l}\text { Irregular } \\
\text { mesh }\end{array}$ & $\begin{array}{c}\text { Adaptive } \\
\text { routing }\end{array}$ & $\begin{array}{l}\text { Power, area } \\
\text { and } \\
\text { performance } \\
\text { efficient; } \\
\text { runtime } \\
\text { adaptivity } \\
\text { for different } \\
\text { applications }\end{array}$ & $\begin{array}{c}\text { HoL } \\
\text { blocking not } \\
\text { addressed; } \\
\text { IP stalling is } \\
\text { not avoided } \\
\text { but reduced }\end{array}$ \\
\hline
\end{tabular}

Physical channels are duplicated in (Michelogiannakis et al 2009) and virtual channels are used in (Michelogiannakis \& Dally 2013; Di Tomaso et al 2012; Ramanujam et al 2011; Jafari et al 2010) to avoid deadlock which leads to area overhead. On the other hand, deadlock is avoided using an adaptive routing algorithm in the proposed work and hence the area overhead is reduced. Performance is limited due to restricted buffering in (Michelogiannakis et al 2009), however, power and area efficiency are gained. In contrast, input channel buffers are employed in each VC in (Michelogiannakis \& Dally 2013; Di Tomaso et al 2012; Ramanujam et al 2011; 
Table 8. Comparison of power and area of the proposed work and that of DSB200 Router (Ramanujam et al 2011).

\begin{tabular}{lcc}
\hline Parameter & Proposed router & $\begin{array}{c}\text { DSB200 router } \\
\text { (Ramanujam } \text { et al 2011) }\end{array}$ \\
\hline Power penalty & 1.36 & 1.39 \\
Area overhead & 1.62 & 1.58 \\
\hline
\end{tabular}

Jafari et al 2010) which lead to performance gains and lessen the power and area efficiency. In the proposed work, we used a small depth of physical buffers in the input channel and buffer depth is dynamically modified by RTBM to handle congestion. As a result, this work gains performance, power and area efficiency.

Regular NoC topologies with a dimension order routing scheme are considered in (Michelogiannakis et al. 2009; Michelogiannakis \& Dally 2013; DiTomaso et al. 2012; Ramanujam et al. 2011; Jafari et al. 2010; Sayed et al. 2012). In MPSoCs, the use of oversized IPs makes the topology irregular and it requires adaptive fault tolerant routing scheme to provide reliable communication. In the proposed work, we considered an irregular mesh topology and applied an adaptive fault tolerant routing algorithm. NoC router architecture shown in figure 3 allows the packets to leave in order with the help of FSM and the arbiter. Hence, the packets are received in order at the destinations contrary to Flexible router in (Sayed et al 2012). NoCs in (Michelogiannakis \& Dally 2013; Di Tomaso et al 2012; Ramanujam et al 2011; Jafari et al 2010; Sayed et al 2012) avoid HoL blocking with the help of virtual channels, but this issue is not addressed in the proposed work. Optimized regulator (Jafari et al 2010) avoids IP stalling with the help of additional buffer in between source IP and interconnect. IP stalling cannot be avoided, but reduced in the proposed work with the help of dynamic buffer allocation and without additional buffering cost.

The comparison of the normalized power and area of the proposed work with respect to the static router and that of DSB200 Router (Ramanujam et al 2011) with respect to their baseline router IBR200 (Ramanujam et al 2011) is presented in table 8. It is shown that the power penalty is slightly lesser and area overhead is more in the proposed router than in the DSB router. The average of saturation throughput improvements of three synthetic traffics, uniform, bit complement and tornado is $18.33 \%$ in the proposed router compared to $18.03 \%$ in DSB200 (Ramanujam et al 2011) router. The overall average latency reduction by the proposed router for realistic traffics (five applications in E3S benchmark) is $22.63 \%$. The overall average latency reduction by DSB200 for realistic traffics (eight traces in SPLASH-2 benchmark) is 17\%. Although DSB200 router outperformed in four real traces of SPLASH-2 benchmark, it has a larger latency in other four real traces compared to IBR200 router. But the performance of our proposed RTBM router is on par with that of the static router at lower loads and it outperforms the static router at higher loads. However, DSB200 router absolutely differs from the proposed router in view of architecture. DSB200 router uses virtual channels which incur additional area overhead.

\section{Conclusion}

In this work, we considered an irregular mesh NoC where some links may be broken or some routers may be absent. An adaptive routing algorithm is used to route the packets reliably to any part of the irregular mesh NoC. The throughput results achieved is the proof of the packets reached their destinations successfully. We also proposed a heterogeneous router in which buffer 
slots are dynamically allocated during runtime using a buffer management algorithm. The proposed router provides a $30.42 \%$ reduction in the average latency and $18.33 \%$ improvement in throughput for synthetic traffic patterns compared to that by the router with static buffer allocation. For E3S benchmark applications, the proposed router offers $22.63 \%$ reduction in average latency. The proposed router utilizes the buffers efficiently. Therefore, it consumes $53 \%$ less power and occupies only half the silicon area compared to the static router buffer depth of 10 to achieve the same performance.

\section{References}

Ahmad B, Ahmadinia A and Arslan T 2008 Dynamically reconfigurable NoC with bus based interface for ease of integration and reduced designed time. In: Proc. NASA/ESA Conf. Adapt. Hardw. Syst. (AHS): 309-314

Ahonen T and Nurmi J 2007 Hierarchically heterogenous network-on-chip. In: Proc. Int. Conf. Comput as a Tool (EUROCON): 2580-2586

Al Faruque M A and Henkel J 2008 Minimizing virtual channel buffer for routers in on-chip communication architectures. In: Proc. Conf. Des., Autom. Test Euro. (DATE): 1238-1243

Al Faruque M A, Ebi T and Henkel J 2008 ROAdNoC: Runtime observability for an adaptive network on chip architecture. In: Proc. IEEE/ACM Int. Conf. Comput.-Aided Des. (ICCAD): 543-548

Benini L and De Mircheli G 2002 Network on chips: A new SoC paradigm. IEEE Comput. 35(1): 70-78

Dally W J and Towles B 2001 Route packets, not wires: On-Chip interconnection networks. In: Proc. 38th Design Automation Conf. (DAC 01), ACM Press: 684-689

Di Tomaso D, Morris R, Kodi A K, Sarathy A and Louri A 2012 Extending the energy efficiency and performance with channel buffers, crossbars, and topology analysis for Network-on-Chips. IEEE Trans. Very Large Scale Integr (VLSI) Syst. 21(11): 2141-2154

Dick R Embedded System Synthesis Benchmark Suites (E3S) http://ziyang.eecs.umich.edu/ dickrp/e3s/

EEMBC Consortium http://www.eembc.org/

Jafari F, Zhonghai Lu, Jantsch A and Yaghmaee M H 2010 Buffer optimization in Network-on-Chip through flow regulation. IEEE Trans. Comput.-Aided Des. Integr. Circuits Syst. 29(12): 1973-1986

Jingcao H, Ogras U Y and Marculescu R 2006 System-level buffer allocation for application-specific networks-on-chip router design. IEEE Trans. Comput.-Aided Des. Integr. Circuits Syst. 25(12): 29192933

Lee S E and Bager Zadeh N 2006 Increasing the throughput of an adaptive router in network-on-chip (NoC). Proc. Int. Conf. Hardw. Softw. Codes. Syst. Synth. 82-87

Matos D, Concatto C, Kreutz M, Kastensmidt F, Carro L and Susin A 2011 Reconfigurable routers for low power and high performance. IEEE Trans. Very Large Scale Integr. (VLSI) Syst. 19: 2045-2057

Michelogiannakis G and Dally W J 2013 Elastic buffer flow control for On-Chip Networks. IEEE Trans. Comput. 62(2): 295-309

Michelogiannakis G, Balfour J and Dally W J 2009 Elastic buffer flow control for On-Chip networks. In: Proc. IEEE 15th Int. Symp. High-Performance Computer Architecture (HPCA '09): 151-162

Nicopoulos C, Park K, Vijaykrishnan D N, Yousif S and Das C 2006 ViChaR: A dynamic virtual channel regulator for network-on-chip routers. In: Proc. 39th Annu. Int. Symp. Microarch. (MICRO): 333-346

Ramanujam R S, Soteriou V, Lin Bill and Peh Li-Shiuan 2011 Extending the effective throughput of NoCs with distributed shared-buffer routers. IEEE Trans. Comput.-Aided Des. Integr. Circuits Syst. 30(4): 548561

Sayed M S, Shalaby A, El-Sayed M and Goulart V 2012 Flexible router architecture for network-on-chip. J. Comput. Math. Appl. 64(5): 1301-1310

Schonwald T, Zimmerman J, Bringmann O and Rosentiel W 2007 Fully adaptive fault-tolerant routing algorithm for Network-on-Chips architectures. Euromicro Conf. on Digital System Design Architectures, Methods and Tools(DSD): 527-534 
Singh A 2005 Load-balanced routing in interconnection networks. PhD thesis in Electrical Eng., Stanford University

Su W, Shen J and Hsiung P 2011 Network-On-Chip router design with buffer stealing. In: Design Automation Conference (ASP-DAC): 160-164

Valinataj M and Mohammadi S 2010 A fault-aware, reconfigurable and adaptive routing algorithm for NoC applications. In: 18th IEEE/IFIP VLSI System On Chip Conference (VLSI-SoC): 13-18

Xuning C and Peh L 2003 Leakage power modeling and optimization in interconnection networks. In: Proc. Int. Symp. Low Power Electron. Des. (ISLPED): 90-95

Ye T T, Benini L and Micheli G De 2002 Analysis of power consumption on switch fabrics in network routers. In: Proc. 39th Des. Autom. Conf. (DAC): 524-529

Yixuan Zhang, Randy Morris Jr. and Avinash K Kodi 2011 Design of a performance enhanced and power reduced dual-crossbar Network-on-Chip (NoC) architecture. Microprocess. Microsyst. 35(2): 110-118

Zhonghai Lu 2005 Using wormhole switching for Networks on Chip: Feasibility analysis and microarchitecture adaptation. Licentiate thesis. Royal Institute of Technology 